\title{
Thermodynamics of a gas of hadrons with attractive and repulsive interactions within an $S$-matrix formalism
}

\author{
Ashutosh Dash, ${ }^{*}$ Subhasis Samanta, ${ }^{\dagger}$ and Bedangadas Mohanty \\ School of Physical Sciences, National Institute of Science Education and Research, HBNI, Jatni 752050, India
}

(Received 8 June 2018; revised manuscript received 23 January 2019; published 29 April 2019)

\begin{abstract}
We report the effect of including repulsive interactions on various thermodynamic observables calculated using an $S$-matrix based hadron resonance gas (HRG) model applied to already available corresponding results with only attractive interactions [A. Dash, S. Samanta, and B. Mohanty, Phys. Rev. C 97, 055208 (2018)]. The attractive part of the interaction is calculated by parametrizing the two-body phase shifts using the $K$ matrix formalism, while the repulsive part is included by fitting to the experimental phase shifts which carry the information about the nature of the interaction. We find that the bulk thermodynamic variables for a gas of hadrons, such as energy density, pressure, entropy density, speed of sound, and specific heat, are suppressed by the inclusion of repulsive interactions and are more pronounced for second- and higher-order correlations and fluctuations, particularly for the observables $\chi_{Q}^{2}, \chi_{B}^{2}-\chi_{B}^{4}$, and $C_{B S}$ in the present model. We find a good agreement between lattice QCD simulations and the present model for $C_{B S}$. We have also computed two leadingorder Fourier coefficients of the imaginary part of the first-order baryonic susceptibility at imaginary baryon chemical potential within this model and compared them with the corresponding lattice results. Additionally, assuming that the value of interacting pressure versus temperature for a gas of hadrons calculated in the $S$-matrix formalism is the same as that from a van der Waals HRG (VDWHRG) model, we have quantified the attractive and repulsive interactions in our model in terms of attractive and repulsive parameters used in the VDWHRG model. The values of parameters thus obtained are $a=1.54 \pm 0.064 \mathrm{GeV} \mathrm{fm}^{3}$ and $r=0.81 \pm 0.014 \mathrm{fm}$.
\end{abstract}

DOI: 10.1103/PhysRevC.99.044919

\section{INTRODUCTION}

One of the primary goals of observing relativistic heavy ion collisions is the study of the QCD (quantum chromodynamics) phase diagram [1]. There are at least two phases in the phase diagram: one where the degrees of freedom are quarks and gluons, called the quark gluon plasma (QGP) phase, and other where the degrees of freedom are hadronic. An approach to study the properties of the hadronic phase formed by hadronization of the QGP is through a statistical model of a gas of hadrons, called the hadron resonance gas (HRG) model [2]. The hadron resonance gas [1,3-13] models have successfully described the hadron multiplicities produced in relativistic nuclear collisions over a wide range of center-of-mass energies. The main result of such an investigation was the observation of a rise in the extracted chemical freeze-out temperature values from lower energies

\footnotetext{
*ashutosh.dash@niser.ac.in

† subhasis.samant@gmail.com

¥bedanga@niser.ac.in; on sabbatical leave to Experimental Physics Department, CERN, CH-1211 Geneva 23, Switzerland.
}

Published by the American Physical Society under the terms of the Creative Commons Attribution 4.0 International license. Further distribution of this work must maintain attribution to the author $(s)$ and the published article's title, journal citation, and DOI. Funded by $S C O A P^{3}$. to almost a constant value of temperature $T \simeq 155-165 \mathrm{MeV}$ at higher energies, supplemented with the decrease of the baryon chemical potential $\left(\mu_{B}\right)$ with increasing energy [14]. The saturation of temperature supports Hagedorn's limiting temperature hypothesis [15], suggesting the possibility of a phase boundary. Similarly, theoretical investigation of QCD on lattice (LQCD) at vanishing $\mu_{B}$ indeed predicts a sharp increase of thermodynamical quantities near deconfinement temperature $T_{c}$ [16-23]. The HRG model is also successful in describing LQCD data related to the bulk properties of hadronic matter in thermal and chemical equilibrium below $T_{c}[17,19,20,22-24]$.

The phenomenal success of the ideal HRG (IDHRG) model in predicting the hadronic yields can be attributed to a theorem by Dashen and Ma [25] which states that the partition function of an interacting hadronic gas can be decomposed into a free and an interacting part. Considering that only resonances contribute to the interacting part, it can be shown that in a narrow resonance width approximation the net effect of the interacting part is equivalent to considering all such hadronic resonances as free particles. However, relaxing the above assumptions by including resonances of finite widths (both overlapping and nonoverlapping), it has been seen that the variation of thermodynamic variables with temperature changes substantially [26-30]. Further, it can be argued that such interactions contribute only to the attractive part of partition function, and the inclusion of a repulsive part could partially negate the effect of the attractive part. For 
example, in Refs. [31-49] the authors have used an excluded volume approach which only had the repulsive part whereas Refs. [50-57] considered a van der Waals (VDW) type of interaction, which has both the attractive and repulsive parts, and a comparison of the calculated thermodynamic pressure from both the approaches shows the feature as discussed above.

In our previous work, Ref. [58], we had developed a HRG model with attractive interactions between hadrons using the $K$-matrix formalism. In the present work, we extend the $K$ matrix formalism to include repulsive interactions between the hadrons using the $S$-matrix formalism. In Ref. [58], we used the $K$-matrix formalism to calculate the phase shifts of the resonance spectral function, in contrast to the popular Breit-Wigner parametrization. It has been argued previously that the $K$-matrix formalism preserves the unitarity of the scattering matrix ( $S$ matrix) and neatly handles multiple resonances $[29,58,59]$. However, the formalism fails to handle any repulsive channel in the scattering matrix. Therefore in this work we include the repulsive part by fitting to experimental phase shifts that encode information about the nature of the interaction. We use the phase shift data from the Scattering Analysis Interactive Database (SAID) partial wave analysis for nucleon-nucleon $(N N)$, pion-nucleon $(\pi N)$, and kaonnucleon $(K N)$ interactions in their respective isospin channels [60-62]. Additionally, we have also included the repulsive isotensor channel in the pion-pion $(\pi \pi)$ scattering, as has been pointed out in many earlier works $[27,63]$.

After constructing the interacting hadron resonance gas model with both attractive and repulsive interactions using phase shift information for various hadronic interactions, we calculate the various thermodynamic observables like pressure, energy density, entropy density, interaction measure, specific heat, speed of sound, and susceptibilities. The temperature dependences of these observables is then compared with corresponding results from lattice QCD, IDHRG, and HRG models with attractive interactions using the $K$-matrix formalism.

The paper is organized in the following manner. In the next section we discuss the formalism used to introduce repulsive interactions to our HRG model developed earlier using the $K$-matrix approach with attractive interactions [58]. In Sec. III we discuss the results from the new interacting HRG model with both attractive and repulsive interactions among the hadrons. The temperature dependence of our results is compared to those from LQCD and IDHRG (with different hadron spectrum). Finally in Sec. IV we summarize our findings.

\section{FORMALISM}

The equation of state for an interacting gas of hadrons of a single species can be computed by using the method of virial expansion. Specifically, the pressure of such a gas can be written as [64]

$$
P(\beta, \mu)=\frac{1}{\beta} \sum_{i=1}^{\infty} \frac{J_{i}}{i !} \xi^{i},
$$

where $\xi=(m / 2 \beta \pi)^{3 / 2} e^{\beta \mu}$, and the inverse temperature, chemical potential, and mass are denoted by $\beta, \mu, m$ respectively. The term $J_{i}$ takes into account the interaction between groups of $i$ hadrons, which are given as

$$
J_{1}=1, \quad J_{2}=\int d V_{2}\left(e^{-\beta U_{12}}-1\right),
$$

etc., where $U_{12}$ is the interaction energy. Differentiating Eq. (1) with respect to $\mu$, we obtain the expression for number density, i.e.,

$$
n(\beta, \mu)=\left(\frac{\partial P}{\partial \mu}\right)_{\beta, V}=\sum_{i=1}^{\infty} \frac{J_{i}}{(i-1) !} \xi^{i} .
$$

Eliminating $\xi$ to the first order from Eqs. (1) and (3) gives us the ideal equation of state $P=n T$, where $T$ is the temperature. For a relativistic noninteracting quantum gas the expression for the pressure is given in [27]. The classical virial equation of state truncated at the second order is given as $P=n T[1+n B(T)]$, where $B(T)=-J_{2} / 2$ is called the second virial coefficient. In this work while calculating the virial coefficients we will be using the $S$-matrix approach to statistical mechanics, which has also been used previously in Refs. [27,65-67] to study the thermodynamics of interacting hadrons.

In the $S$-matrix formalism, the second virial coefficient is related to the scattering amplitude or alternatively to the scattering phase shifts $\delta_{l}^{I}$ for a given spin $l$ and isospin $I$ channel. The correction to the ideal pressure for binary interactions between particles of species $i$ with particles of species $j$ is given as

$$
\begin{aligned}
P_{\mathrm{int}}^{i j} & =\frac{T J_{2}}{2} z_{i} z_{j} \\
& =\frac{z_{i} z_{j}}{2 \pi^{3} \beta^{2}} \int_{M_{i j}}^{\infty} d \varepsilon \varepsilon^{2} K_{2}(\beta \varepsilon) \sum_{I, l}^{\prime} g_{I, l} \frac{\partial \delta_{l}^{I}(\varepsilon)}{\partial \varepsilon},
\end{aligned}
$$

where the terms $z_{i}, g_{I, l}$, and $\varepsilon$ stand for the fugacity, the spin-isospin degeneracy factor, and the total center-of-mass energy respectively. The function $K_{2}(x)$ stands for the modified Bessel function of the second kind and the term $M_{i j}$ is the invariant mass of the interacting hadron pair $i j$ at threshold. Additionally, there is a sum over all possible spin-isospin channels and the prime over the summation sign denotes that for given $l$, the sum over $I$ is restricted to values consistent with statistics. A similar expression for interacting part of number density can also be derived such that $n_{\text {int }}^{i j}=\beta P_{\text {int }}^{i j}$ for $i \neq j$ and $n_{\text {int }}^{i}=2 \beta P_{\text {int }}^{i}$ for $i=j$.

The total pressure and number density for an interacting system containing $N$ such hadronic species is then given as

$$
\begin{aligned}
& P=\sum_{i}^{N} P_{\mathrm{id}}^{i}+\sum_{i, j \geqslant i}^{N} P_{\text {int }}^{i j}=P_{\text {id }}+P_{\text {int }}, \\
& n=\sum_{i}^{N} n_{\text {id }}^{i}+\sum_{i, j \geqslant i}^{N} n_{\text {int }}^{i j}=n_{\text {id }}+n_{\text {int }},
\end{aligned}
$$

where $P_{\mathrm{id}}^{i}, n_{\mathrm{id}}^{i}$ are the ideal contributions of the species $i$ to pressure and number density of the system respectively. In the 
present work, the contribution to the non interacting (ideal) part comes from all the stable hadrons. An equation of state truncated to the second order can be derived as in the previous paragraph such that the total pressure $P$ or the total number density $n$ is given as

$$
\begin{aligned}
& P=n T-P_{\text {int }}\left(\frac{n}{n_{\text {id }}}\right)^{2}=n T+\bar{P}_{\text {int }}, \\
& n=\frac{P}{T}+\frac{n_{\text {int }}}{2}\left(\frac{P}{P_{\text {id }}}\right)^{2}=\frac{P}{T}+\bar{n}_{\text {int }},
\end{aligned}
$$

where $\bar{P}_{\text {int }}$ and $\bar{n}_{\text {int }}$ are the effective contributions of interaction to pressure and number density respectively.

From Eq. (4), it can be seen that the second virial coefficient gives a positive (attractive) or negative (repulsive) contribution depending on whether the derivatives of phase shifts are positive or negative. The phase shifts are obtained from experiments or from theoretical calculations. In the present work, we determine the attractive phase shifts using the $K$-matrix formalism, which takes the masses and partial widths of resonances from the PDG (Particle Data Group) [68] as input. Since the $K$-matrix formalism is not applicable for handling the repulsive phase shifts, these are obtained by fitting to experimental data. We would like to note here that since we do not have the information of masses and widths of resonances (mentioned in PDG) that decay into a pair of nucleons, we extract phase shifts in such situations by fitting to experimental data.

\section{A. $K$-matrix formalism}

A theoretical way of calculating phase shifts is to use the $K$-matrix formalism. The $K$-matrix formalism preserves the unitarity of the $S$ matrix and neatly handles multiple resonances [59]. In addition to that, widths of the resonances are handled naturally in the above formalism. The notion of an ideal HRG is only valid for narrow resonances and not for broad resonances; in contrast, the $K$-matrix formalism can be applied quite generally. Similarly, for overlapping resonances the $K$-matrix gives a more accurate description of the phase shifts than the Breit-Wigner parametrization. In Ref. [29] the $K$-matrix formalism was used to study an interacting gas of hadrons, and it was extended further in [58].

The resonances contributing to the process $a b \rightarrow R \rightarrow c d$ appear as a sum of poles in the $K$ matrix,

$$
K_{a b \rightarrow c d}=\sum_{R} \frac{g_{R \rightarrow a b}(\sqrt{s}) g_{R \rightarrow c d}(\sqrt{s})}{m_{R}^{2}-s},
$$

where $a, b$ and $c, d$ are hadrons and the sum on $R$ runs over the number of resonances with mass $m_{R}$. The sum is restricted to the addition of resonances for a given spin $l$ and isospin $I$. The residue functions are given by

$$
g_{R \rightarrow a b}^{2}(\sqrt{s})=m_{R} \Gamma_{R \rightarrow a b}(\sqrt{s}),
$$

where $\sqrt{s}$ is the center-of-mass energy and $\Gamma_{R \rightarrow a b}(\sqrt{s})$ is the energy dependent partial decay width, i.e., the total width times the branching ratio for the channel $R \rightarrow a b$, given as [59]

$$
\Gamma_{R \rightarrow a b}(\sqrt{s})=\Gamma_{R \rightarrow a b}^{0} \frac{m_{R}}{\sqrt{s}} \frac{q_{a b}}{q_{a b 0}}\left[B^{l}\left(q_{a b}, q_{a b 0}\right)\right]^{2} .
$$

The momentum $q_{a b}$ is given as

$$
q_{a b}(\sqrt{s})=\frac{1}{2 \sqrt{s}} \sqrt{\left(s-\left(m_{a}+m_{b}\right)^{2}\right)\left(s-\left(m_{a}-m_{b}\right)^{2}\right)},
$$

where $m_{a}$ and $m_{b}$ are the masses of decaying hadrons $a$ and $b$.

In Eq. (11), $q_{a b 0}=q_{a b}\left(m_{R}\right)$ is the resonance momentum at $\sqrt{s}=m_{R}$, and $\Gamma_{R}^{0}$ is the partial width of the pole at half maximum for the channel $R \rightarrow a b$. The $B^{l}\left(q_{a b}, q_{a b 0}\right)$ are the Blatt-Weisskopf barrier factors, which can be expressed in terms of momentum $q_{a b}$ and resonance momentum $q_{a b 0}$ for the orbital angular momentum $l$. The detailed expression for $B^{l}\left(q_{a b}, q_{a b 0}\right)$ can be found in Ref. [59].

Furthermore, once one computes the $K$ matrix by providing the relevant masses and widths of resonances, the phase shift can be obtained using the relation

$$
\delta_{l}^{I}=\tan ^{-1} K(\sqrt{s}) .
$$

Here we would like to note that a comparison between the empirical phase shifts of resonances and the $K$-matrix approach gives almost identical results for resonances like $\rho(770), K^{*}(892), N(1680)$, etc.

\section{B. Experimental phase shifts}

As mentioned earlier, for repulsive interactions and for interactions where the information about $m_{R}$ and $\Gamma_{R}$ are not available, the $K$-matrix formalism is not applicable and we resort to extraction of phase shifts from experimental data. In our extraction of repulsive $(\pi N, K N)$ and nucleon-nucleon $(N N)$ interaction phase shifts, we use the data from the SM16 partial wave analysis [60]. For the repulsive isotensor channel $\delta_{0}^{2}$ in the $\pi-\pi$ scattering, we use the data from Ref. [69]. However, the $S$-matrix formalism elucidated here is only applicable for elastic scattering, and the inelastic part that enters into the analysis by fitting to experimental data has to be removed. To get around this problem, we make an estimate of the contribution coming from the inelastic part by first defining a generic $l$ dependent scattering amplitude $f_{l}(\sqrt{s})$ :

$$
f_{l}(\sqrt{s})=\frac{\eta_{l} e^{2 i \delta_{l}}-1}{2 i},
$$

where $\eta_{l}$ is the inelastic parameter. The elastic cross section is given as

$$
\sigma_{\mathrm{el}}=\frac{4 \pi}{q^{2}} \sum_{l}(2 l+1) \sin ^{2} \delta_{l},
$$

and the inelastic cross section is given by

$$
\sigma_{\text {inel }}=\frac{\pi}{q^{2}} \sum_{l}(2 l+1)\left(1-\eta_{l}^{2}\right),
$$

where $q$ is the center-of-mass momentum. The total cross section $\sigma$ is the sum of Eqs. (15) and (16). We can approximate the contribution to the elastic part of the phase shift $\delta_{\mathrm{el}}$ by the 

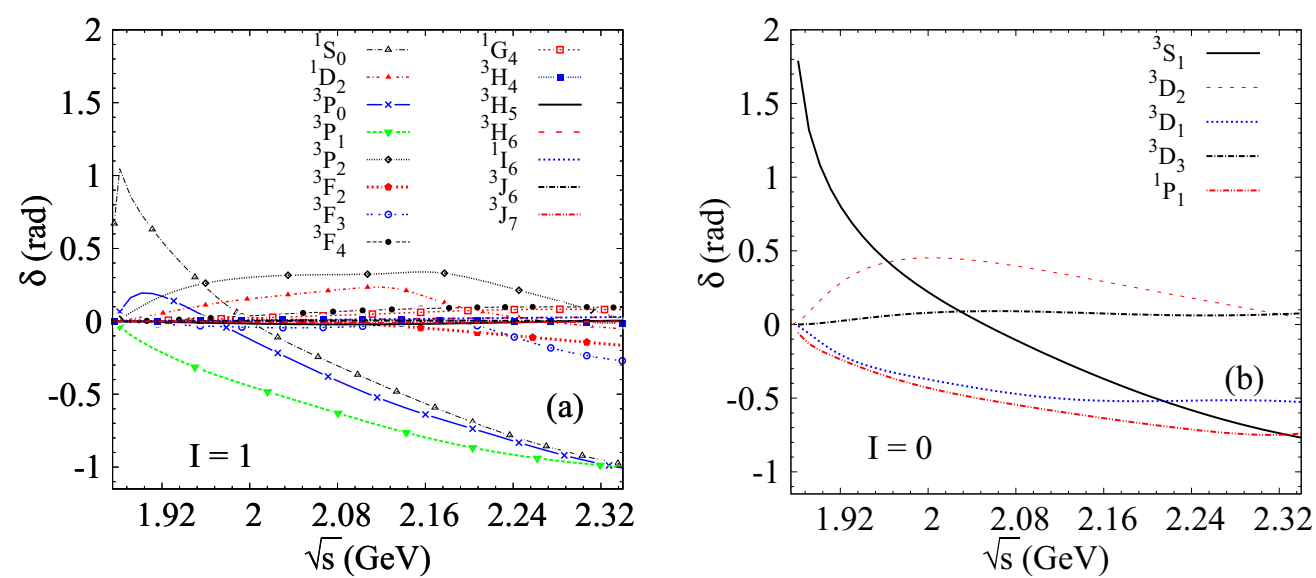

FIG. 1. Energy dependence of $N N$ scattering phase shifts taken from SAID partial-wave analysis [60]. The notation to specify $N N$ scattering channels is ${ }^{2 S+1} l_{J}$, where $l, S, J$ correspond to orbital, spin, and total angular momentum respectively.

following expression:

$$
\delta_{\mathrm{el}} \approx \sin ^{-1}\left(\sqrt{\frac{\sigma_{\mathrm{el}}}{\sigma}} \sin \delta\right)
$$

where $\delta$ is the total phase shift that is obtained from the fit to experimental data [60-62].

\section{1. $N$ interactions}

For the nucleon-nucleon $(N N)$ interaction we have included the phase shifts for $l \leqslant 7$ in both $I=0$ and $I=1$ isospin channels. Combinations of $l, S$, and $I$ are chosen so that the total wave function for $N N$ interaction is antisymmetric as dictated by Pauli's principle. We have restricted the range of energies up to the pion $(\pi)$ production threshold. Beyond this threshold, the contribution from the inelastic channels become dominant and the present formalism fails to disentangle the contribution from the elastic and inelastic parts. However, below this threshold, where the contribution from inelasticities are subdominant, we can extract the contribution from the elastic part using the approximation (17). In order to use Eq. (17) we need a parametrization of the cross section as a function of energy, which in the present study is taken from Ref. [70]:

$$
\sigma^{N N}(\mathrm{mb})= \begin{cases}23.5+1000\left(p_{\text {lab }}-0.7\right)^{4}, & p_{\text {lab }}<0.8 \mathrm{GeV}, \\ 23.5+\frac{24.6}{1+\exp \left(-\frac{P_{\text {lab }}-1.2}{0.10}\right)}, & 0.8<p_{\text {lab }}<5 \mathrm{GeV}, \\ 41+60\left(p_{\text {lab }}-0.9\right) \exp \left(-1.2 p_{\text {lab }}\right), & 1.5<p_{\text {lab }}<5 \mathrm{GeV},\end{cases}
$$

where $p_{\text {lab }}$ is the laboratory momentum. Similarly the elastic cross section $\sigma_{\mathrm{el}}$ can be parametrized as

$$
\sigma_{\mathrm{el}}^{N N}(\mathrm{mb})= \begin{cases}23.5+1000\left(p_{\mathrm{lab}}-0.7\right)^{4}, & p_{\mathrm{lab}}<0.8 \mathrm{GeV}, \\ \frac{1250}{p_{\mathrm{lab}}+50}-4\left(p_{\mathrm{lab}}-1.3\right)^{2}, & 0.8<p_{\mathrm{lab}}<2 \mathrm{GeV}, \\ \frac{77}{p_{\mathrm{lab}}+1.5}, & p_{\mathrm{lab}}>2 \mathrm{GeV}\end{cases}
$$

By comparing Eqs. (18) and (19), we can see that the contribution from inelastic processes is small below $p_{\text {lab }}<0.8 \mathrm{GeV}$

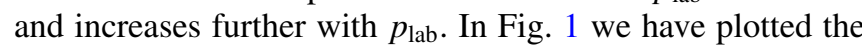
experimental $N N$ phase shifts from the SAID partial-wave analysis [60] as a function of center-of-mass energy $(\sqrt{s})$. The dominant contribution comes from lower $l$ values, e.g., the ${ }^{1} S_{0}$ phase shift which peaks at lower $\sqrt{s}$ and then falls sharply or the rapidly falling and largely repulsive ${ }^{3} S_{1}$ phase shift. An interesting case to observe is the triplet $P$ waves, which can have $J=0,1,2$ corresponding to phase shifts ${ }^{3} P_{0},{ }^{3} P_{1},{ }^{3} P_{2}$. The behaviors of the phase shifts are quite different in the above three channels: from zero crossing to purely repulsive and purely attractive cases as seen in Fig. 1. This could be attributed to the spin-orbit coupling, which splits them into triplet states having different behavior depending on the sign and strength of the coupling. However, most of the phase shifts become negative at higher $\sqrt{s}$, signifying the hard core nature of $N N$ interaction. We would like to note that for $N N$ interaction the contribution from bound states, e.g., the ${ }^{3} S_{1}$ channel, which forms deuteron at threshold, is not taken into account in Eq. (4).

\section{2. $\pi N$ interactions}

For pion-nucleon $(\pi N)$ interaction we have included only those phase shifts [61] which are purely repulsive, and the attractive parts are from the $K$-matrix parametrization. Here, we have restricted the energies to the eta $(\eta)$ production threshold, 
and the cross sections are parametrized from Ref. [70] using the isobar model as

$$
\sigma^{\pi N}(\mathrm{mb})=\frac{326.5}{1+4\left(\frac{\sqrt{s}-1.215}{0.110}\right)^{2}} \frac{q^{3}}{q^{3}+(0.18)^{3}},
$$

where $q$ is the center-of-mass momentum. In the range of momenta $0.5<p_{\text {lab }}<1.5 \mathrm{GeV}$, the inelastic channel $\pi N \rightarrow$ $\pi \pi N$ is the most dominant whose cross section can be parametrized as

$$
\sigma_{\text {inel }}^{\pi N}(\mathrm{mb})=74\left(p_{\text {lab }}-0.555\right)^{2} p_{\text {lab }}^{-4.04} \mathrm{GeV} .
$$

The dominant repulsive contribution in the $\pi N$ interaction comes from the $S_{31}\left(l_{2 I, 2 J}\right)$ phase shift corresponding to the $\Delta(1620)$ resonance. We would like to stress here that in our previous study of resonances in Ref. [58], using the $K$-matrix formalism, resonances such as $\Delta(1620), \Delta(1910)$, $\Delta(1930)$, and $N(1720)$ were included in the attractive part of the $S$-matrix, via their masses and partial decay widths (i.e., branching fraction times the total width) for a resonance $R$ interacting through the process $a b \rightarrow R \rightarrow a b$, where $a$ and $b$ are the corresponding hadrons. However, a comparison to experimental phase shifts through the factor $\partial \delta^{l}(\epsilon) / \partial \epsilon$ has shown that it is negative below the $\eta$ production threshold. Thus, such resonances are included in the repulsive part by fitting to experimental phase shifts.

\section{3. $K \boldsymbol{N}$ interactions}

For the $K N$ interaction, the dominant repulsive contribution comes from the $S_{11}\left(l_{I, 2 J}\right)$ phase shift containing the $\Sigma(1660)$ resonance. Similar to the case of $\pi N, \Sigma$ resonances like $\Sigma(1660), \Sigma(1750)$, and $\Sigma(1915)$ and $\Lambda$ resonances like $\Lambda(1520), \Lambda(1600)$, and $\Lambda(1690)$ were considered attractive in [58], but here we include them in the repulsive part, since $\partial \delta^{l}(\epsilon) / \partial \epsilon$ is negative below the inelastic production threshold [62]. The cross sections are parametrized from Ref. [71] as

$$
\begin{aligned}
\sigma^{K N}(\mathrm{mb}) & =23.91+17.0 \exp \left(-\frac{\left(p_{\text {lab }}-10\right)^{2}}{0.12}\right), \\
p_{\text {lab }} & <2.5 \mathrm{GeV} \text { and } \\
\sigma_{\mathrm{el}}^{K N}(\mathrm{mb}) & =172.38 \exp \left[-2.0\left(p_{\text {lab }}+0.1\right)\right], \\
p_{\text {lab }} & <0.7 \mathrm{GeV} .
\end{aligned}
$$

\section{4. $\pi-\pi$ interactions}

For the pion-pion $(\pi \pi)$ interaction we have included the dominant repulsive phase shift from Ref. [69], in the isotensor channel $\delta_{0}^{2}$, as do previous studies [27,63]. This phase shift is known to cancel the isoscalar channel $\delta_{0}^{0}$ containing the broad $f_{0}(500)$ ( $\sigma$ meson). The relevant energies have been restricted to the pion production threshold.

\section{RESULT}

In Fig. 2, we show the temperature variation at zero chemical potential for various thermodynamic quantities such as scaled pressure, energy density, entropy density, speed of sound, and the specific heat capacity at constant volume. Results of the attractive $K$-matrix (KM) based HRG model from Ref. [58] are compared with the total contribution (Total), which contains both attractive and repulsive channels, obtained in the present work. In Ref. [58] it was found that the effect of attractive interaction through the KM approach increases the value of all thermodynamic observables compared to the ideal HRG result (IDHRG 1). It must be noted that the $K$-matrix formalism includes only those resonances which have a two-body decay mode and only these resonances were included in IDHRG 1 . We observe that the effect of repulsive interactions cancels some of the contributions from attractive channels, thereby slightly lowering the net result for "Total" relative to KM for the observables studied here. A second comparison with the ideal HRG model, Fig. 2(a), that considers all the confirmed hadrons and resonances consisting of up, down, and strange flavor valence quarks listed in the PDG 2016 Review [68] [IDHRG (PDG 2016)], shows a better agreement with lattice data. However, it is worth mentioning here that the agreement of "IDHRG (PDG 2016)" with the LQCD data is because of the increase in the number of degeneracies and not due to some inherent interaction that is naturally present in the system revealed within the $S$-matrix formalism. On the whole, we conclude that the effects of repulsive channels suppress the bulk variables studied here, compared to the $K$-matrix (KM) approach, and these are shown in Fig. 2.

Similar to thermodynamic observables, it was found in Ref. [58] that the $K$-matrix formalism leads to an increment in the values of diagonal and off-diagonal susceptibilities compared to the ideal HRG result. The effects of repulsive interactions are most prominent when we calculate these second-order diagonal and off-diagonal susceptibilities. Results for $\chi_{B}^{2}, \chi_{Q}^{2}, \chi_{B S}^{11}$, and $\chi_{B Q}^{11}(B, Q$ stand for baryon and electric charge respectively; the definition of susceptibilities can be found in Ref. [58]) shown in Fig. 3 agree better with the LQCD data in the case when both attraction and repulsion are taken into account than in the $K$-matrix formalism. The effect of repulsion is mostly visible in the baryonic sector. For example, we have checked for $\chi_{B}^{2}$ that the contribution from repulsive interaction has the following order: $\pi N>K N>$ $N N$. Although we find that more channels are repulsive in $N N$ interaction than in $\pi N$ interaction, the effect of repulsion on observables like $\chi_{B}^{2}$ is more from $\pi N$ interaction. This is because the effect of repulsion in elastic $\pi N$ interaction is dominant in the energy range $1.07<\sqrt{s}<1.67 \mathrm{GeV}$, while for elastic $N N$ interaction it is in the range $1.88<\sqrt{s}<2.34$ $\mathrm{GeV}$. This fact is reflected when we compute thermodynamic observables in the relevant temperature ranges. We have also checked for the remaining second-order diagonal and ofdiagonal susceptibilities, and the difference between "Total" and the $K$-matrix formalism for $\chi_{S}^{2}$ is small and for $\chi_{Q S}^{11}$ it is negligible.

Lattice observables like fluctuations and correlations of conserved charges at finite net baryon density are expected to be sensitive to the modeling of baryonic interactions. However, lattice calculation at finite $\mu_{B}$ is not possible because of the sign problem. Methods like Taylor series expansion and analytic continuation from imaginary have been devised to get around this problem [72-83]. Following Ref. [84], we directly compare the predictions of the $S$-matrix formalism to lattice 

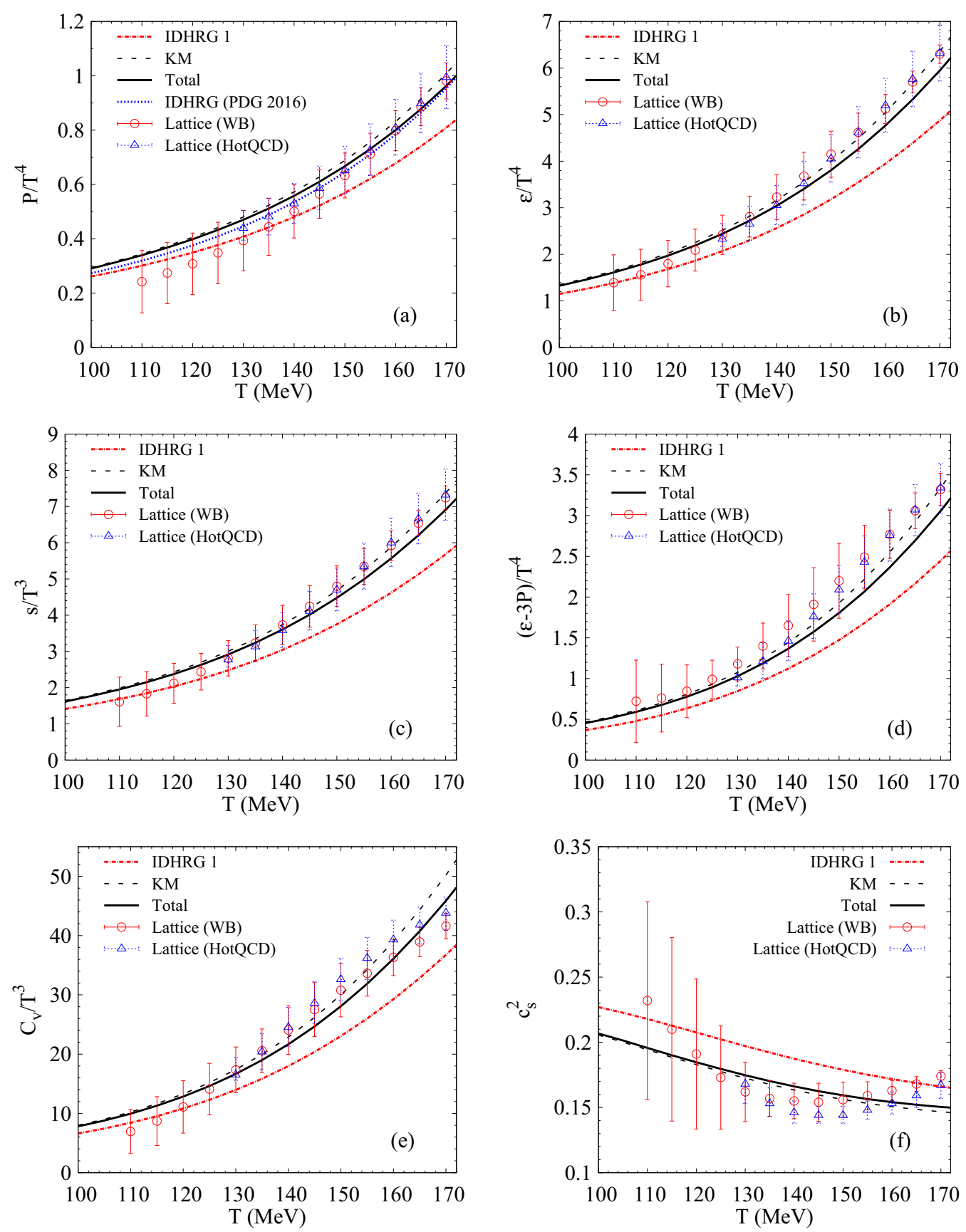

FIG. 2. Temperature dependence of various thermodynamic quantities $\left[\left(\right.\right.$ a) $P / T^{4}$, (b) $\varepsilon / T^{4}$, (c) $s / T^{3}$, (d) $\left(\varepsilon-3 P\right.$ ) $/ T^{4}$, (e) $C_{V} / T^{3}$, and (f) $c_{S}^{2}$ ] at zero chemical potential. "Total" contains both the attractive and repulsive interactions whereas KM contains only the attractive part. IDHRG 1 corresponds to results of the ideal HRG model, with same number of particles as used in the KM formalism. IDHRG (PDG 2016) in (a) corresponds to results of the ideal HRG model for all the hadrons and resonances listed in PDG 2016 [68]. Results are compared with lattice QCD data of Refs. [21] (WB) and [22] (HotQCD).

data at imaginary chemical potential instead of performing analytic continuation to real chemical potential. Since the QCD pressure is an even function of real $\mu_{B}$, the first-order net baryon susceptibility assuming Maxwell-Boltzmann (MB) statistics can be written as [84]

$$
\chi_{B}^{1}=\sum_{j=1}^{\infty} b_{j} \sinh \left(j \mu_{B} / T\right)
$$

where $b_{j}$ contains the information from different baryonic sectors. Using analytic continuation, one can convert the above sum to a Fourier series expansion where the Fourier coefficients are given as

$$
b_{j}(T)=\frac{2}{\pi} \int_{0}^{\pi} d x \operatorname{Im}\left[\chi_{B}^{1}\left(T, i \mu_{B}\right)\right] \sin (j x),
$$

where $x=\mu_{B} / T$. 

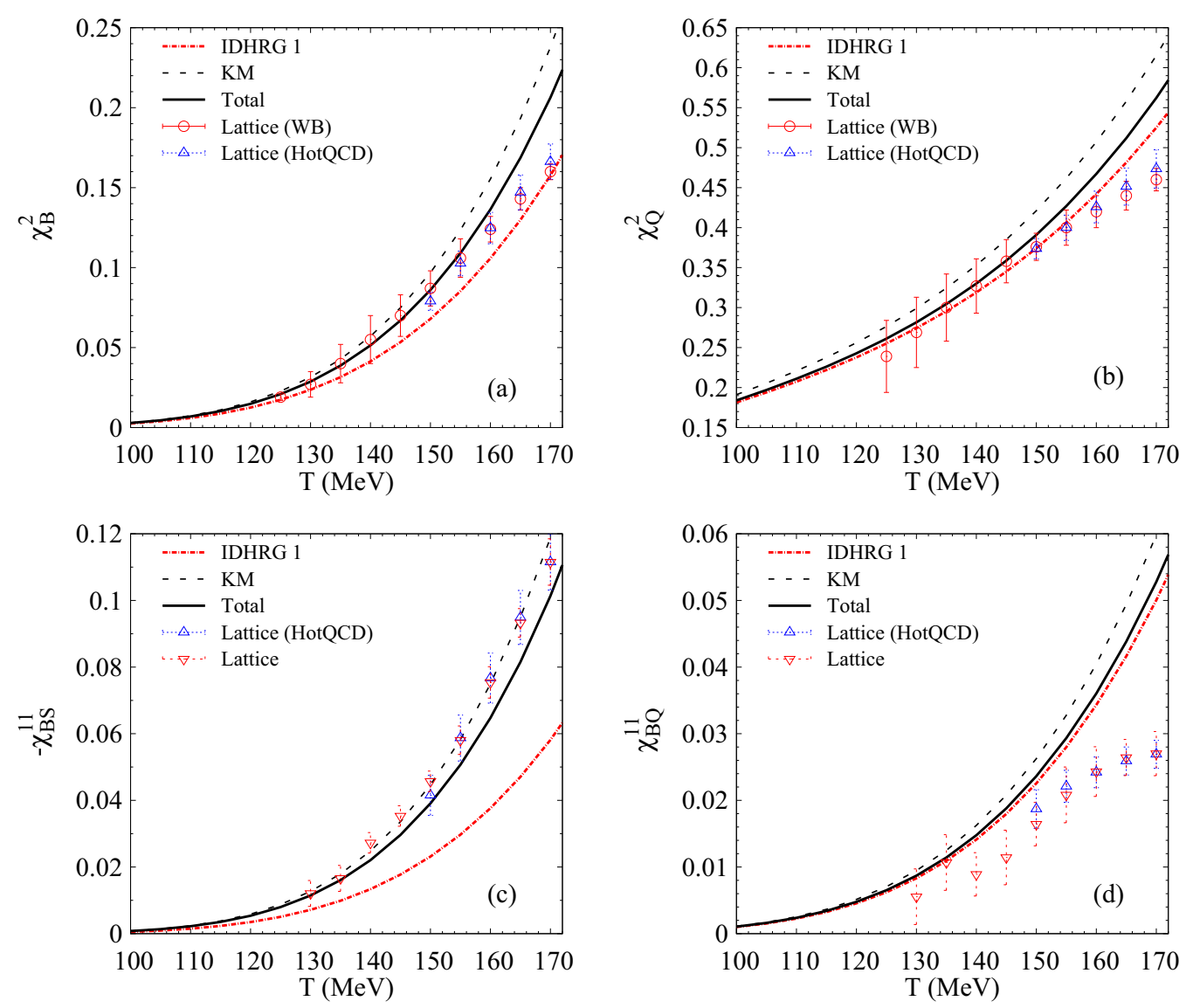

FIG. 3. Temperature dependence of secondorder susceptibilities $\left[(a) \chi_{B}^{2}\right.$, (b) $\chi_{Q}^{2}$, (c) $\chi_{B S}^{11}$, and (d) $\left.\chi_{B Q}^{11}\right]$ at zero chemical potential. "Total" contains both the attractive and repulsive interactions whereas KM contains only the attractive part. IDHRG 1 corresponds to results of the ideal HRG model, with same number of particles as used in the KM formalism. Results are compared with lattice QCD data of Refs. [17] (WB), [19] (HotQCD), and [23] (Lattice).

The results of two leading-order Fourier coefficients $b_{1}(T), b_{2}(T)$ computed using the $S$-matrix formalism are compared to lattice results and are shown in Fig. 4(a). We find a very good agreement between the coefficient $b_{1}(T)$ and lattice QCD results using imaginary chemical potential. Moreover, we found that $b_{2}(T)$ is quite small compared to the lattice results, which is due to the inclusion of only $N N$ interaction and not other baryon-baryon interactions. However, we found that $b_{2}(T)$ is positive for $T<135 \mathrm{MeV}$ and negative above this temperature, contrary to the results of Ref. [84] which are negative throughout the temperature range. This can be understood from the isospin weighted sum of phase shifts of $N N$ interaction, which is positive for small $\sqrt{s}$ and falls rapidly at large $\sqrt{s}$, showing the hard core nature of $N N$ interaction at short distances.

The contribution from interaction can be explored further by considering certain combinations of diagonal and of-diagonal susceptibilities which are identically zero for a noninteracting or ideal HRG but not for a noninteracting gas of quarks and gluons [85]. The quantity $\chi_{B}^{2}-\chi_{B}^{4}=0$ for a hadron gas which has baryon number \pm 1 , but not for a noninteracting QGP for which $\chi_{B}^{2}-\chi_{B}^{4}>0$, since all quarks carry a baryon number of $\pm 1 / 3$. However, for an interacting gas, the inclusion of $N N$ interaction which carries a net baryon number \pm 2 might give us a nonzero result. It is particularly instructive to note that this observable is related to Fourier coefficients such that $\chi_{B}^{2}-\chi_{B}^{4}=-\sum_{j=2}^{j=\infty} j\left(j^{2}-1\right) b_{j}(T)$, assuming MB statistics. In our case, since the contribution comes only from the term $j=2$ for $N N$ interaction, we have $\chi_{B}^{2}-\chi_{B}^{4}=-6 b_{2}(T)$. We compare this with the $S$-matrix formalism where the ideal part is computed assuming FermiDirac (FD) statistics. This is shown in Fig. 4(b), and the result shows that $-6 b_{2}(T)$ changes sign in accordance to the discussion in the previous paragraph. However, we find that the influence of statistics (FD) in the $S$-matrix formalism leads to an increase in the value of observable $\chi_{B}^{2}-\chi_{B}^{4}$ and shifts the change in sign to a lower temperature. The above observation is in agreement with lattice data, which also shows a similar change in sign when moving from lower to higher temperature. For temperatures $T>110 \mathrm{MeV}$ we find that $\chi_{B}^{2}-\chi_{B}^{4}>0$, again indicating the hard core nature of $N N$ interaction. In Ref. [54,67] the same increasing trend of $\chi_{B}^{2}-$ $\chi_{B}^{4}$ with temperature was also found using a repulsive mean field in a multicomponent hadron gas and excluded volume approach. Our results using the $S$-matrix formalism validate the previous results. Moreover, one should note that the effect of including only $N N$ interaction is rather small compared to the results obtained by Ref. [54,67] which can be improved 

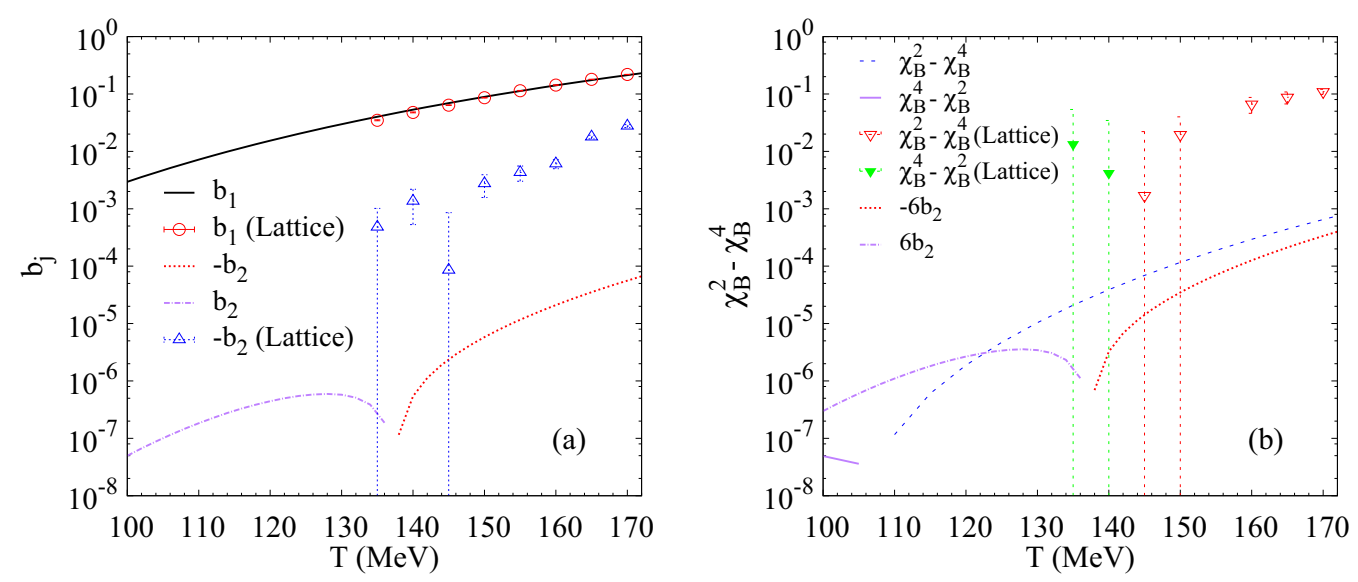

FIG. 4. The left panel shows the variation of Fourier coefficients $b_{j}(T)$ with temperature, computed using the $S$-matrix formalism, compared to the lattice results from Ref. [84]. Open symbols (circles and triangles) denote the result from lattice QCD. Solid (black) line denotes the result of Fourier coefficient $b_{1}(T)$. The dot-dashed purple line and the dotted red line represent the positive and negative parts of the Fourier coefficient $b_{2}(T)$ respectively. The right panel shows the variation of $\chi_{B}^{2}-\chi_{B}^{4}$ with temperature at zero chemical potential. Nonzero values come mainly due the $N N$ interaction, which is shown by the dashed blue and solid purple lines, denoting the positive and negative parts assuming FD statistics in the ideal part. The dot-dashed purple line and the dotted red line represent the positive and negative parts of the Fourier coefficient $6 b_{2}(T)$ respectively (see text). Results are compared with lattice QCD data of Ref. [23] (Lattice), with open red and solid green symbols denoting the positive and negative parts.

upon adding other baryon-baryon interactions in the partition function. However, we do not have information about the experimental phase shifts of other baryon-baryon interactions, and one has to substitute chiral effective theory [86,87] or other such methods, which is left as a future work. Other observables like $v_{1}=\chi_{B S}^{31}-\chi_{B S}^{11}$ and $v_{2}=1 / 3\left(\chi_{S}^{2}-\chi_{S}^{4}\right)-$ $2 \chi_{B S}^{13}-4 \chi_{B S}^{22}-2 \chi_{B S}^{31}$ [85] are trivially zero in our analysis since we do not have information about interactions (phase shifts) among baryons which have $|B|>1$ and $|S|=1$ or vice versa.

The correlation between the strangeness $S$ and baryon number $B$ is a sensitive probe of the relevant microscopic degrees of freedom. The quantity $C_{B S}$ [88], defined as $C_{B S}=$ $-3 \chi_{B S}^{11} / \chi_{S}^{2}$, is one such observable. For a gas of noninteracting QGP $C_{B S}=1$, but for a gas of hadrons dominated by kaons and antikaons [a light quark is always correlated with its strange partner (kaons) or vice versa (antikaons)] $C_{B S}<1$. On the other hand, a system dominated by strange baryons which correlate light quark (antiquark) with strange quark (antiquark) hence has $C_{B S}>1$. Therefore, for large baryon chemical potential, $C_{B S}$ could be larger than unity in a hadron gas. Moreover, a significant difference between LQCD and the ideal HRG model was reported previously [89]. It has been argued that such a discrepancy can be cured by allowing additional strange hadrons which have not been confirmed but are predicted in various quark models [83,90]. Figure 5 shows that the difference between LQCD and ideal HRG can be accounted for by including interaction without invoking any additional hadrons.

We match the second virial coefficient obtained using the $S$-matrix formalism with the virial coefficient $B(T)$ of a van der Waals gas and extract the VDW parameters $a$ and $b$. For a VDW gas the coefficient $B(T)$ is given as [64]

$$
B(T)=b-\frac{a}{T}
$$

where $b=16 \pi r^{3} / 3$, where $r$ is the hard core radius and $a$ is a positive constant denoting attraction. Thus, the interacting pressure $P_{\text {int }}^{\mathrm{VDW}}$ is related to the number density $n^{\mathrm{VDW}}$ for a VDW equation of state as

$$
P_{\mathrm{int}}^{\mathrm{VDW}}=-\left(n^{\mathrm{VDW}}\right)^{2} T B(T),
$$

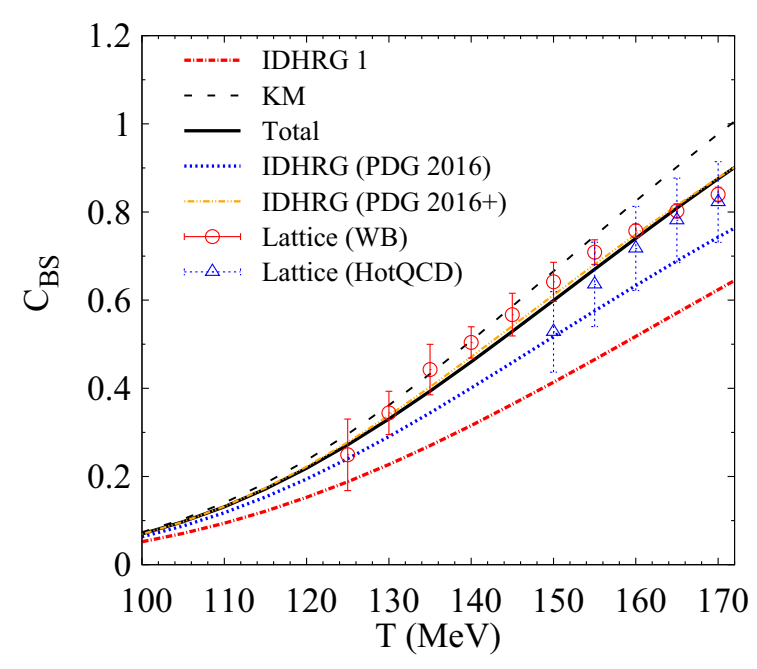

FIG. 5. The temperature dependence of $C_{B S}$ at zero chemical potential calculated in the current work (Total). IDHRG 1 corresponds to results of the ideal HRG model, with the same number of particles as used in the KM or $S$-matrix formalism. IDHRG (PDG 2016) corresponds to results of the ideal HRG model using the hadronic spectrum of PDG 2016 [68]. Results of the ideal HRG model with additional resonances which are not yet confirmed are also shown [IDHRG (PDG 2016+)]. Lattice QCD data of $C_{B S}$ are taken from Refs. [17] (WB) and [19] (HotQCD). 


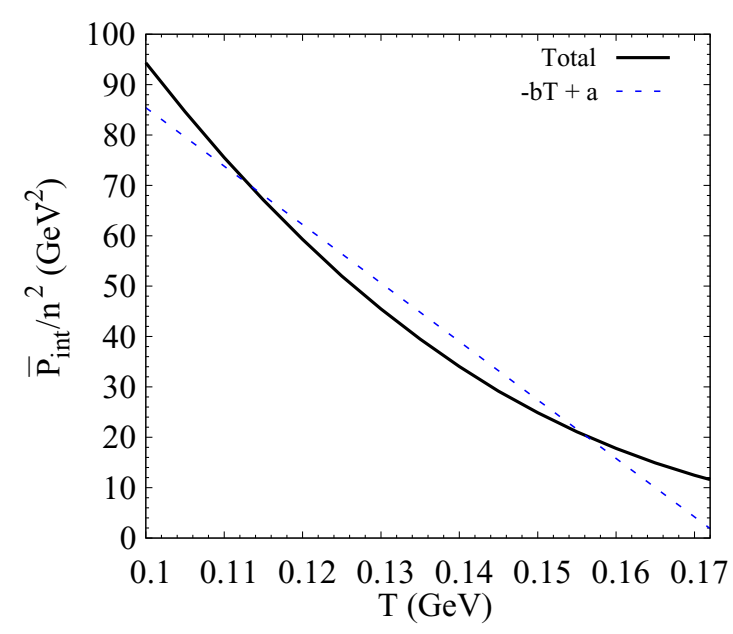

FIG. 6. Variation of $\bar{P}_{\text {int }} / n^{2}$ with temperature at zero chemical potential. "Total" corresponds to both the attractive and repulsive interactions in the HRG model from the current work. The curve is fitted with the straight line $-T B(T)=-b T+a$ [see Eq. (25)].

Matching $P_{\text {int }}^{\mathrm{VDW}}$ with $\bar{P}_{\text {int }}$, i.e., the second term of Eq. (7), and $n^{\mathrm{VDW}}$ with Eq. (8), we extract the values of $a$ and $b$. Figure 6 shows the $\bar{P}_{\text {int }} / n^{2}$ calculated as a function of temperature using the $S$-matrix formalism compared to that of an interacting VDW gas. In the case of a temperature independent VDW parameters, the curve in Fig. 6 would be a straight line. This study indicates that the simple (constant) parametrization of the VDW parameters is not correct in a realistic situation, where both the attractive parameter $a$ and the repulsive parameter $b$ could in general be temperature dependent. This fact also supports models [27], where a temperature dependent radius was used. However, assuming the VDW parameters are temperature independent, a straight line fit to the results in Fig. 6 with a functional form of $-b T+a$ is carried out to extract the VDW parameters. The values of the VDW parameters are $a=1.54 \pm 0.064 \mathrm{GeV} \mathrm{fm}^{3}$ and the hard core radius $r=0.81 \pm 0.014 \mathrm{fm}$. We would like to comment here that the extracted parameters can be seen as some effective values containing contributions from meson-meson, meson-nucleon, and nucleon-nucleon interactions averaged over many hadronic species, while Refs. [51,57] extracted these parameters considering only baryon-baryon interaction.

\section{SUMMARY}

To summarize, we have included repulsive interaction between hadrons by fitting to experimental phase shifts which carry information about the nature of the interaction. The attractive part of the interaction is also included and was calculated by parametrizing the two-body phase shifts using the $K$-matrix formalism [58], which is known to preserve the unitarity of the $S$-matrix. Since the experimental phase shifts for the attractive part of the interactions were available for the $N N$ scattering, those are used in calculations. Thermodynamic quantities like pressure, energy density, trace anomaly, specific heat, speed of sound, etc. were calculated using the $S$-matrix formalism. The results indicate that the effect of repulsive channels is to suppress the bulk variables studied here. This finding suggests that in contrast to certain channels like $\pi-\pi$ interaction, where the isospin-weighted sum of $s$ wave attractive and repulsive phase shifts cancel each other, we found that this observation is not true for all channels. We find that although some partial cancellation is occurring among various phase shifts in $\pi N, K N$, and $N N$ interaction channels, the resultant interaction is substantial and far from exact cancellation.

Similarly, we compared the Fourier coefficients using the $S$-matrix formalism with lattice data at imaginary chemical potential. The leading-order coefficient $b_{1}(T)$ reproduces lattice data, while the next-to-leading order coefficient $b_{2}(T)$ is smaller than the prediction of lattice QCD data. However, we found that $b_{2}(T)$ is positive for $T<135 \mathrm{MeV}$ and negative for $T>135 \mathrm{MeV}$, contrary to Ref. [84], where it is negative throughout the temperature range. This can be attributed to the isospin weighted degeneracy of $N N$ interaction that is positive at lower $\sqrt{s}$ and negative at higher $\sqrt{s}$.

We found that the most prominent effect of repulsive interactions is seen when we calculate the second- and higherorder fluctuations and correlation. The inclusion of repulsive interaction leads to a better agreement of observables like $\chi_{B}^{2}$ and $\chi_{Q}^{2}$ with lattice data than the result of only attractive interaction considered in Ref. [58] using the $K$-matrix formalism. This is because, in addition to other attractive interactions as considered in [58], resonances like $\Delta(1620), \Delta(1910)$ in the $\pi N$ interaction and like $\Sigma(1660)$ in the $K N$ interaction were considered attractive in the $K$-matrix formalism. But here, we have included such resonances in the repulsive part. This is understood as a comparison to the experimental phase shifts of such resonances through the factor $\partial \delta^{l}(\epsilon) / \partial \epsilon$ has shown that it is negative and hence repulsive. Here, we would like to note that the strength from different channels to the repulsive part of the second virial coefficient is in the order such that $\pi N>K N>N N$.

Particularly, the two most interesting observations which resulted from the current work are as follows. First, we find that the observable $\chi_{B}^{2}-\chi_{B}^{4}>0$ for temperatures $T>$ $110 \mathrm{MeV}$, in an interacting HRG model discussed in this work, is contrary to the expectation $\chi_{B}^{2}-\chi_{B}^{4}=0$ for an uncorrelated gas of hadrons like in the IDHRG model. We also observed that statistics (FD or MB) play a crucial role on the values and the sign of this observable. However, the effect of interaction is only from $N N$ interaction, which is rather small compared to the results obtained by Refs. [54,67]. The present result can be viewed as a first attempt to address such observables in a model which does not have any free parameters compared to previous works. This result can be improved by adding other baryon-baryon interactions using information from chiral effective theory, etc. Second, for the observable $C_{B S}$, which is a sensitive probe of the relevant microscopic degrees of freedom of a system, the HRG model in the present formalism very well describes the LQCD data. It is also seen from Fig. 5 that the IDHRG model with additional strange hadronsm which has not yet been confirmed, agrees with the LQCD data at a similar level [89]. The difference in physics interpretation is the following: the IDHRG model with additional strange hadrons attributes the matching of LQCD data relative to 
normal IDHRG model to the increase in hadronic degrees of freedom for the system of hadrons. Our results, in contrast, attribute the matching toto interactions among the constituents that is captured naturally through the formalism used in the current work.

Finally we have tried to quantify the attractive and repulsive interactions in our model in terms of the VDWHRG attractive and repulsive parameters $a$ and $r$, respectively. In doing so we assume that the parameter values do not change with temperature and the interacting parts of the pressure are same in the two models at a given temperature. It may be noted that our results as shown in Fig. 6 indicate that $a$ and $r$ could be temperature dependent. It would be interesting to calculate various transport co-efficients in a $S$-matrix based HRG model and compare to other different types of HRG models and corresponding lattice QCD results.

\section{ACKNOWLEDGMENTS}

B.M. acknowledges financial support from a J. C. Bose National Fellowship of Department of Science and Technology (DST), Government of India. S.S. and A.D. thank Volodymyr Vovchenko for helpful discussion and acknowledge financial support from Department of Atomic Energy (DAE), Government of India.
[1] A. Andronic, P. Braun-Munzinger, and J. Stachel, Phys. Lett. B 673, 142 (2009).

[2] F. Becattini, in International School on Quark-Gluon Plasma and Heavy Ion Collisions: Past, Present, Future, Villa Gualino, Torino, Italy, December 8-14, 2008, arXiv:0901.3643.

[3] P. Braun-Munzinger, J. Stachel, J. P. Wessels, and N. Xu, Phys. Lett. B 344, 43 (1995).

[4] J. Cleymans, D. Elliott, H. Satz, and R. L. Thews, Z. Phys. C 74, 319 (1997).

[5] G. D. Yen and M. I. Gorenstein, Phys. Rev. C 59, 2788 (1999).

[6] P. Braun-Munzinger, I. Heppe, and J. Stachel, Phys. Lett. B $\mathbf{4 6 5}$, 15 (1999).

[7] J. Cleymans and K. Redlich, Phys. Rev. C 60, 054908 (1999).

[8] F. Becattini, J. Cleymans, A. Keranen, E. Suhonen, and K. Redlich, Phys. Rev. C 64, 024901 (2001).

[9] P. Braun-Munzinger, D. Magestro, K. Redlich, and J. Stachel, Phys. Lett. B 518, 41 (2001).

[10] F. Becattini, J. Manninen, and M. Gazdzicki, Phys. Rev. C 73, 044905 (2006).

[11] A. Andronic, P. Braun-Munzinger, and J. Stachel, Nucl. Phys. A 772, 167 (2006).

[12] S. Das, D. Mishra, S. Chatterjee, and B. Mohanty, Phys. Rev. C 95, 014912 (2017).

[13] A. Andronic, P. Braun-Munzinger, K. Redlich, and J. Stachel, Nature (London) 561, 321 (2018).

[14] P. Braun-Munzinger and J. Stachel, Nature (London) 448, 302 (2007).

[15] R. Hagedorn, Nuovo Cimento Suppl. 3, 147 (1965).

[16] Y. Aoki, G. Endrodi, Z. Fodor, S. D. Katz, and K. K. Szabo, Nature (London) 443, 675 (2006).

[17] S. Borsanyi, Z. Fodor, S. D. Katz, S. Krieg, C. Ratti, and K. Szabo, J. High Energy Phys. 01 (2012) 138.

[18] S. Gupta, X. Luo, B. Mohanty, H. G. Ritter, and N. Xu, Science 332, 1525 (2011).

[19] A. Bazavov et al. (HotQCD Collaboration), Phys. Rev. D 86, 034509 (2012).

[20] R. Bellwied, S. Borsanyi, Z. Fodor, S. D. Katz, and C. Ratti, Phys. Rev. Lett. 111, 202302 (2013).

[21] S. Borsanyi, Z. Fodor, C. Hoelbling, S. D. Katz, S. Krieg, and K. K. Szabo, Phys. Lett. B 730, 99 (2014).

[22] A. Bazavov et al. (HotQCD Collaboration), Phys. Rev. D 90, 094503 (2014).

[23] R. Bellwied, S. Borsanyi, Z. Fodor, S. D. Katz, A. Pasztor, C. Ratti, and K. K. Szabo, Phys. Rev. D 92, 114505 (2015).
[24] F. Karsch, K. Redlich, and A. Tawfik, Phys. Lett. B 571, 67 (2003).

[25] R. Dashen, S.-K. Ma, and H. J. Bernstein, Phys. Rev. 187, 345 (1969).

[26] M. I. Gorenstein, M.-S. Tsai, and S.-N. Yang, Phys. Rev. C 51, 1465 (1995).

[27] R. Venugopalan and M. Prakash, Nucl. Phys. A 546, 718 (1992).

[28] W. Weinhold, B. Friman, and W. Norenberg, Phys. Lett. B 433, 236 (1998).

[29] A. Wiranata, V. Koch, M. Prakash, and X. N. Wang, Phys. Rev. C 88, 044917 (2013).

[30] P. Huovinen, P. M. Lo, M. Marczenko, K. Morita, K. Redlich, and C. Sasaki, Phys. Lett. B 769, 509 (2017).

[31] D. H. Rischke, M. I. Gorenstein, H. Stoecker, and W. Greiner, Z. Phys. C 51, 485 (1991).

[32] J. Cleymans, M. I. Gorenstein, J. Stalnacke, and E. Suhonen, Phys. Scr. 48, 277 (1993).

[33] G. D. Yen, M. I. Gorenstein, W. Greiner, and S.-N. Yang, Phys. Rev. C 56, 2210 (1997).

[34] S. K. Tiwari, P. K. Srivastava, and C. P. Singh, Phys. Rev. C 85, 014908 (2012).

[35] V. V. Begun, M. Gazdzicki, and M. I. Gorenstein, Phys. Rev. C 88, 024902 (2013).

[36] A. Andronic, P. Braun-Munzinger, J. Stachel, and M. Winn, Phys. Lett. B 718, 80 (2012).

[37] J. Fu, Phys. Lett. B 722, 144 (2013).

[38] A. Bhattacharyya, S. Das, S. K. Ghosh, R. Ray, and S. Samanta, Phys. Rev. C 90, 034909 (2014).

[39] M. Albright, J. Kapusta, and C. Young, Phys. Rev. C 90, 024915 (2014).

[40] V. Vovchenko, D. V. Anchishkin, and M. I. Gorenstein, Phys. Rev. C 91, 024905 (2015).

[41] M. Albright, J. Kapusta, and C. Young, Phys. Rev. C 92, 044904 (2015)

[42] G. P. Kadam and H. Mishra, Phys. Rev. C 92, 035203 (2015).

[43] J. Kapusta, M. Albright, and C. Young, Eur. Phys. J. A 52, 250 (2016).

[44] L. M. Satarov, V. Vovchenko, P. Alba, M. I. Gorenstein, and H. Stoecker, Phys. Rev. C 95, 024902 (2017).

[45] V. Vovchenko and H. Stoecker, Phys. Rev. C 95, 044904 (2017).

[46] R. P. Adak, S. Das, S. K. Ghosh, R. Ray, and S. Samanta, Phys. Rev. C 96, 014902 (2017).

[47] P. Alba, W. M. Alberico, A. Nada, M. Panero, and H. Stöcker, Phys. Rev. D 95, 094511 (2017). 
[48] P. Alba, V. Vovchenko, M. I. Gorenstein, and H. Stoecker, Nucl. Phys. A 974, 22 (2018).

[49] V. Vovchenko, M. I. Gorenstein, and H. Stoecker, Phys. Rev. C 98, 064909 (2018).

[50] V. Vovchenko, D. V. Anchishkin, and M. I. Gorenstein, J. Phys. A 48, 305001 (2015).

[51] V. Vovchenko, D. V. Anchishkin, and M. I. Gorenstein, Phys. Rev. C 91, 064314 (2015).

[52] V. Vovchenko, D. V. Anchishkin, M. I. Gorenstein, and R. V. Poberezhnyuk, Phys. Rev. C 92, 054901 (2015).

[53] K. Redlich and K. Zalewski, Acta Phys. Pol. B 47, 1943 (2016).

[54] V. Vovchenko, M. I. Gorenstein, and H. Stoecker, Phys. Rev. Lett. 118, 182301 (2017).

[55] V. Vovchenko, Phys. Rev. C 96, 015206 (2017).

[56] V. Vovchenko, A. Motornenko, P. Alba, M. I. Gorenstein, L. M. Satarov, and H. Stoecker, Phys. Rev. C 96, 045202 (2017).

[57] S. Samanta and B. Mohanty, Phys. Rev. C 97, 015201 (2018).

[58] A. Dash, S. Samanta, and B. Mohanty, Phys. Rev. C 97, 055208 (2018).

[59] S. U. Chung, J. Brose, R. Hackmann, E. Klempt, S. Spanier, and C. Strassburger, Ann. Phys. (NY) 4, 404 (1995).

[60] R. L. Workman, W. J. Briscoe, and I. I. Strakovsky, Phys. Rev. C 94, 065203 (2016).

[61] R. L. Workman, R. A. Arndt, W. J. Briscoe, M. W. Paris, and I. I. Strakovsky, Phys. Rev. C 86, 035202 (2012).

[62] J. S. Hyslop, R. A. Arndt, L. D. Roper, and R. L. Workman, Phys. Rev. D 46, 961 (1992).

[63] W. Broniowski, F. Giacosa, and V. Begun, Phys. Rev. C 92, 034905 (2015).

[64] L. D. Landau and E. M. Lifshitz, Statistical Physics, Part 1, Course of Theoretical Physics, Vol. 5 (Butterworth-Heinemann, Oxford, 1980).

[65] P. M. Lo, B. Friman, K. Redlich, and C. Sasaki, Phys. Lett. B 778, 454 (2018).

[66] P. M. Lo, B. Friman, M. Marczenko, K. Redlich, and C. Sasaki, Phys. Rev. C 96, 015207 (2017).

[67] P. Huovinen and P. Petreczky, Phys. Lett. B 777, 125 (2018).

[68] C. Patrignani et al. ( Particle Data Group), Chin. Phys. C 40, 100001 (2016).
[69] R. Garcia-Martin, R. Kaminski, J. R. Pelaez, J. R. de Elvira, and F. J. Yndurain, Phys. Rev. D 83, 074004 (2011).

[70] J. Cugnon, J. Vandermeulen, and D. L'Hote, Nucl. Instrum. Methods Phys. Res., Sect. B 111, 215 (1996).

[71] B. Hong, K. M. Maung, J. W. Wilson, and W. W. Buck, NASA STI/Recon Technical Report No. 89, 1989 (unpublished).

[72] C. R. Allton, S. Ejiri, S. J. Hands, O. Kaczmarek, F. Karsch, E. Laermann, C. Schmidt, and L. Scorzato, Phys. Rev. D 66, 074507 (2002).

[73] S. Basak et al. (MILC Collaboration), Proceedings, 26th International Symposium on Lattice Field Theory (Lattice 2008), Williamsburg, VA, July 14-19, 2008 [PoS LATTICE 2008, 171 (2008)].

[74] R. V. Gavai and S. Gupta, Phys. Rev. D 78, 114503 (2008).

[75] P. de Forcrand and O. Philipsen, Nucl. Phys. B 642, 290 (2002).

[76] M. D'Elia and M.-P. Lombardo, Phys. Rev. D 67, 014505 (2003).

[77] L.-K. Wu, X.-Q. Luo, and H.-S. Chen, Phys. Rev. D 76, 034505 (2007).

[78] P. de Forcrand and O. Philipsen, J. High Energy Phys. 11 (2008) 012.

[79] M. D’Elia and F. Sanfilippo, Phys. Rev. D 80, 014502 (2009).

[80] O. Philipsen and C. Pinke, Phys. Rev. D 89, 094504 (2014).

[81] C. Czaban, F. Cuteri, O. Philipsen, C. Pinke, and A. Sciarra, Phys. Rev. D 93, 054507 (2016).

[82] M. D'Elia, G. Gagliardi, and F. Sanfilippo, Phys. Rev. D 95, 094503 (2017).

[83] P. Alba et al., Phys. Rev. D 96, 034517 (2017).

[84] V. Vovchenko, A. Pasztor, Z. Fodor, S. D. Katz, and H. Stoecker, Phys. Lett. B 775, 71 (2017).

[85] A. Bazavov et al., Phys. Rev. Lett. 111, 082301 (2013).

[86] H. Polinder, J. Haidenbauer, and U.-G. Meissner, Nucl. Phys. A 779, 244 (2006).

[87] J. Haidenbauer, S. Petschauer, N. Kaiser, U. G. Meissner, A. Nogga, and W. Weise, Nucl. Phys. A 915, 24 (2013).

[88] V. Koch, A. Majumder, and J. Randrup, Phys. Rev. Lett. 95, 182301 (2005).

[89] A. Bazavov et al., Phys. Rev. Lett. 113, 072001 (2014).

[90] S. Chatterjee, D. Mishra, B. Mohanty, and S. Samanta, Phys. Rev. C 96, 054907 (2017). 